\section{P270 IDENTIFICATION OF RESPONDER GROUPS TO FLUTICASONE FUROATE/VILANTEROL (FF/VI) IN THE SALFORD LUNG STUDY IN COPD (SLS COPD) USING A CLUSTER ANALYSIS MODEL}

${ }^{1} \mathrm{~A}$ Nicholls, ${ }^{2} \mathrm{~N}$ Diar Bakerly, 'S Collier, ${ }^{1} \mathrm{H}$ Dickinson, 'D Leather, 'I Boucot. 'GSK, Brentford, UK; ${ }^{2}$ Salford Royal NHS Foundation Trust, Salford, UK and Manchester Academic Health Sciences Centre, The University of Manchester, Manchester, UK

\subsection{6/thoraxjnl-2017-210983.412}

Background Identifying patients who respond more favourably to specific therapy allows for optimal disease management (maximising treatment benefits; minimising treatment-related risks) and better allocation of limited healthcare resources. SLS COPD, a 12 month, open-label, randomised controlled trial conducted in UK primary care, compared the clinical effectiveness and safety of initiating once-daily inhaled FF/VI $100 / 25 \mu \mathrm{g}$ versus continuing usual care (UC) in patients with COPD. The trial met its primary effectiveness endpoint demonstrating an $8.4 \%$ reduction in the mean annual rate of moderate/severe exacerbations with $\mathrm{FF} / \mathrm{VI}$ versus UC (95\% CI: $1.1-15.2 ; \mathrm{p}=0.02$; primary effectiveness analysis population). ${ }^{1}$ Aim Identify patient subgroups demonstrating an enhanced response with FF/VI versus UC in SLS COPD, using a cluster analysis approach.

Methods This exploratory post-hoc analysis utilised a datadriven recursive partitioning algorithm (SIDES ${ }^{2}$ ) to identify several candidate patient subgroups, each with the potential to demonstrate added benefit of FF/VI versus UC compared with the parent intent-to-treat (ITT) population, based on the primary effectiveness endpoint. Twenty-four distinct patient variables were considered, including baseline demographics, COPD history and disease characteristics, comorbidities, socioeconomic status and treatment adherence. Following identification of a "best" candidate subgroup, the primary effectiveness model used in the original SLS COPD study ${ }^{1}$ was repeated to evaluate the potential additional benefit of FF/VI versus UC in this subgroup.

Results Eight candidate subgroups were identified, defined by combinations of coronary artery disease (CAD) diagnosis, $\mathrm{CAT}^{\mathrm{TM}}$ score, age and polypharmacy. The subgroup indicating the greatest potential treatment effect of initiating FF/VI versus continuing UC comprised 1430/2799 (51\%) ITT patients with no CAD diagnosis, baseline CAT score $\leq 33$ and age $\geq 61$ years. In this subgroup, the mean annual rate of moderate/ severe exacerbations was reduced by $21.40 \% \quad(95 \%$ CI: 12.79-29.17) with FF/VI versus UC, contrasting with the observed reduction of $8.4 \%$ (95\% CI: $1.4-14.9)$ in the overall ITT population. ${ }^{1}$

Conclusions The identified patient subgroup demonstrated an enhanced response with FF/VI versus UC compared to the overall SLS COPD population. Work is ongoing to validate/ confirm these findings in an alternative COPD dataset.

Funding GSK (HZC115151/NCT01551758).

Please refer to page A260 for declarations of interest in relation to abstract P270.

\section{REFERENCES}

1. Vestbo J et al. NEJM 2016;375:1253-60.

2. Lipkovich I et al. Stat Med 2011;30:2601-21.

\section{P271 \\ EFFECT OF EXTRAFINE SINGLE INHALER TRIPLE THERAPY ON LUNG FUNCTION AND USE OF RESCUE MEDICATION: RESULTS FROM THE TRINITY STUDY}

${ }^{1} \mathrm{M}$ Scuri, ${ }^{2} \mathrm{~J}$ Vestbo, ${ }^{3} \mathrm{~A}$ Papi, ${ }^{4} \mathrm{M}$ Corradi, ${ }^{1} \mathrm{I}$ Montagna, ${ }^{1} \mathrm{C}$ Francisco, ${ }^{1} \mathrm{G}$ Cohuet, ${ }^{1} \mathrm{~S}$ Vezzoli, ${ }^{1}$ A Muraro, ${ }^{1} \mathrm{~S}$ Petruzzelli, ${ }^{2} \mathrm{D}$ Singh. ${ }^{1}$ Chiesi Farmaceutici S.p.A, Parma, Italy; ${ }^{2}$ University of Manchester, Manchester, UK; ${ }^{3}$ University of Ferrara, Ferrara, Italy; ${ }^{4}$ University of Parma, Parma, Italy

\subsection{6/thoraxjnl-2017-210983.413}

Rationale Treatment with extrafine triple therapy in a single inhaler has beneficial effects compared to LAMA monotherapy on lung function and symptoms. This analysis focuses on rescue medication use (as this is associated with symptoms) and lung function responder analysis identifying clinically relevant effects.

Methods In this 52 week multicentre, randomised, doubleblind, active-controlled study, 2691 patients with severe to very severe COPD, exacerbations history, and CAT total score $\geq 10$ were randomised $(2: 2: 1)$ to tiotropium, fixed triple (beclometasone/formoterol/glycopyrronium), or free triple (beclometasone/formoterol+tiotropium). Secondary endpoints included FEV1 responders at week 26 and 52 using different thresholds for response and change from baseline in average use of rescue medication.

Results Both fixed and free triple FEV1 responder percentages were significantly greater than tiotropium at weeks 26 and 52 regardless of the threshold used to define the response ( $p<0.001$ for all analyses). At 26 weeks the proportion of responders were $48.0 \%$ (fixed triple) and $48.1 \%$ (free triple) for the $50 \mathrm{ml}$ threshold, and $36.7 \%$ and $34.8 \%$ at the higher $120 \mathrm{~mL}$ threshold, with similar Results at week 52. Corresponding FEV1 responder percentages for tiotropium were lower at the $50 \mathrm{~mL}$ threshold $(35.7 \%$ and $34.8 \%$, at weeks 26 and 52 respectively) and $120 \mathrm{~mL}$ threshold $(25.3 \%$ and $24.8 \%$, respectively). In terms of average percentage of days without rescue medication use over 52 weeks, all treatments showed statistically significant increases from baseline which were more marked with fixed and free triple $(13.9$ [95\%CI: $12 ; 15.8]$ and $14.8 \%$ [95\%CI: $12.1 ; 17.4]$ respectively) compared to $5.2 \%$ [95\% CI: 3.3;7.1] for tiotropium alone $(\mathrm{p}<0.001)$ and no difference observed between fixed and free triple with an adjusted mean difference of $-0.8 \%$ [95\% CI: $-4.1 ; 2.4](p=0.616)$. Average use of rescue medication with both fixed and free triple treatments over 52 weeks compared to tiotropium alone was reduced by 0.6 [95\% CI: $0.4 ; 0.7]$ and 0.6 [95\%CI: 0.5;0.8] puffs/day, respectively $(\mathrm{p}<0.001)$.

Conclusions Extrafine triple therapy in a single inhaler provides superior clinical benefits in severe to very severe COPD patients in terms of lung function (by individual responder analysis) and rescue medication use compared with tiotropium alone.

Please refer to page A260 for declarations of interest in relation to abstract P271. 